\title{
Catalysis
}

\section{Reusable and Flexible Heterogeneous Catalyst for Reduction of TNT by Pd Nanocube Decorated ZnO Nanolayers onto Electrospun Polymeric Nanofibers}

\author{
Osman Arslan, ${ }^{*[a, c]}$ Hamit Eren, ${ }^{*[a]}$ Necmi Biyikli, ${ }^{*[b]}$ and Tamer Uyar ${ }^{*[a]}$
}

An effective method for the fabrication of well designed nanocomposite for the catalytic reduction of 2,4,6-trinitrotoluene (TNT) was developed. Here, cubic palladium (Pd) nanoparticles were utilized for enhancing the interface properties, attachment quality, catalytic yield and stability after the catalysis reactions. Ligand controlled facet growth by the $\mathrm{Br}$ anions during thermal decomposition of the palladium-precursor resulted with cubic shaped average $\sim 13 \mathrm{~nm}$ palladium nanocubes ( $\mathrm{Pd} \mathrm{NC}$ ). The anisotropic $\mathrm{Pd} \mathrm{NC}$ were utilized to decorate the surface of the zinc oxide $(\mathrm{ZnO})$ nanolayers deposited by atomic layer deposition (ALD) technique on the electrospun polyacrylonitrile (PAN) nanofibers. Due to the polymeric nature of the electrospun PAN nanofibers, Pd NC

\section{Introduction}

2,4,6-Trinitrotoluene (TNT) is a widely known chemical explosive which is utilized in mines, bombs and grenades causing a residual contamination in the soil and groundwater. Due to its hazardous effects, remediation of the environment or water sources from TNT needs to be realized by detecting, controlling, and destroying/neutralizing it instantly. Recent efforts generally focus on the detection methods by sensors, chemical noses or electrochemically rather than its elimination. ${ }^{[1]}$ However, an appropriate and sufficient exploration for the decontamination process is still a challenging pose. ${ }^{[1]}$ Therefore, in this study, we focused on fast and effective catalytic reduction

[a] Prof. O. Arslan, H. Eren, Prof. T. Uyar

Institute of Materials Science \& Nanotechnology

UNAM-National Nanotechnology Research Center

Bilkent University

06800, Ankara, TURKEY

E-mail: arslan@unam.bilkent.edu.tr hamit.eren@bilkent.edu.tr uyar@unam.bilkent.edu.tr

[b] Prof. N. Biyikli

Electrical and Computer Engineering

University of Connecticut

Storrs, CT 06269-4157, USA

E-mail: nbiyikli@gmail.com

[c] Prof. O. Arslan

Istanbul Sabahattin Zaim University

Food Engineering Department

Halkali St. No: 2, 34303 Halkali-Kucukcekmece, Istanbul, TURKEY

E-mail: o.arslan@izu.edu.tr

Supporting information for this article is available on the WWW under https://doi.org/10.1002/slct.201701329 decorated nanoweb is highly flexible and has a high surface area. For the sustainable Pd NC decoration on the $\mathrm{ZnO}$ surfaces coated on PAN nanofibers, anchor points were formed by the functional thiol groups which can facilitate the Pd NC attachment and stability on the $\mathrm{ZnO}$ surface. The $-\mathrm{OH}$ and alkyl thiol groups obtained via sol-gel reactions positioned on the $\mathrm{ZnO}$ layer providing a better interface between $\mathrm{ZnO}$ and $\mathrm{Pd} \mathrm{NC}$ which cannot be obtained by pristine PAN nanofibers. Additionally, due to the increased surface interaction, geometrical positioning on fibers for a better intermediate complex formation and stability via soft-soft interaction, Pd NC decorated flexible polymeric electrospun nanoweb provided en hanced catalytic reduction of TNT in aqueous medium.

of TNT by palladium nanocube (Pd NC) decorated $\mathrm{ZnO}$ nanolayers on electrospun polymeric nanofibers. For this purpose, an efficient nanocomposite architecture was designed by choosing a flexible and high surface area polymeric nanofibrous support. The polymeric nanofibers (polyacrylonitrile (PAN)) were obtained by electrospinning method. Then $\mathrm{ZnO}$ nanolayer was coated on PAN nanofibers via atomic layer deposition (ALD) and finally palladium nanocubes ( $P d N C$ ) was decorated onto this $\mathrm{ZnO}$ nanolayer (Figure 1). Since catalytic reduction systems require clean and stable surfaces of $\mathrm{Pd}$ nanostructures, this high surface area architecture was designed for an efficient catalytic reduction of TNT. By sol-gel reactions, anchor points (alkyl thiol) can be formed onto $\mathrm{ZnO}$ nanolayer (Figure 1c) and Pd NC can be easily decorated onto $\mathrm{ZnO}$ coated PAN nanofibers (Figure 1d). This nanocomposite provided fast and effective catalytic reduction of TNT due to high surface area and increased contact between the $\mathrm{Pd}$ nanocube and $\mathrm{ZnO}$ surface together with stability after the catalysis reactions.

Recently, we have shown fabrication of various polymericinorganic core-shell nanofibers by the combination of electrospinning and ALD method. ${ }^{[2-6]}$ In order to deposit $\mathrm{ZnO}$ coatings with controlled thickness and optical properties, exposure times of the organometallic zinc precursor and water, purge durations, exposure-mode diffusion time and substrate temperature can be tuned in the ALD process. This is necessary for a controlled crystal structure, surface functionality and defect states for $\mathrm{ZnO}$ coating. Additionally, selecting the type of polymeric fibers which will be conformally ALD-coated with $\mathrm{ZnO}$ is quite critical. Due to its heat resistance, durability, 


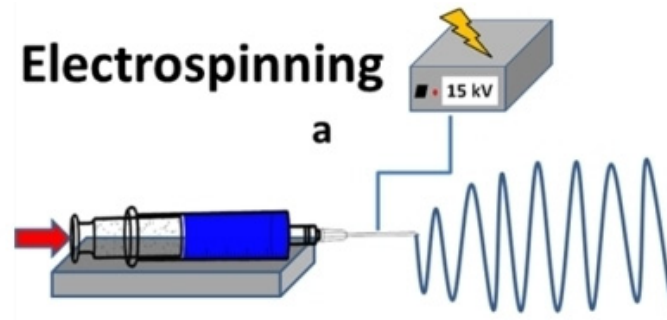

ALD Deposition

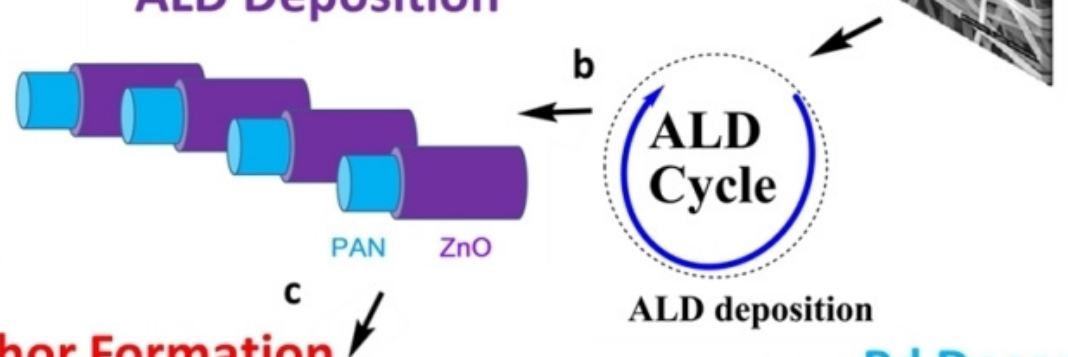

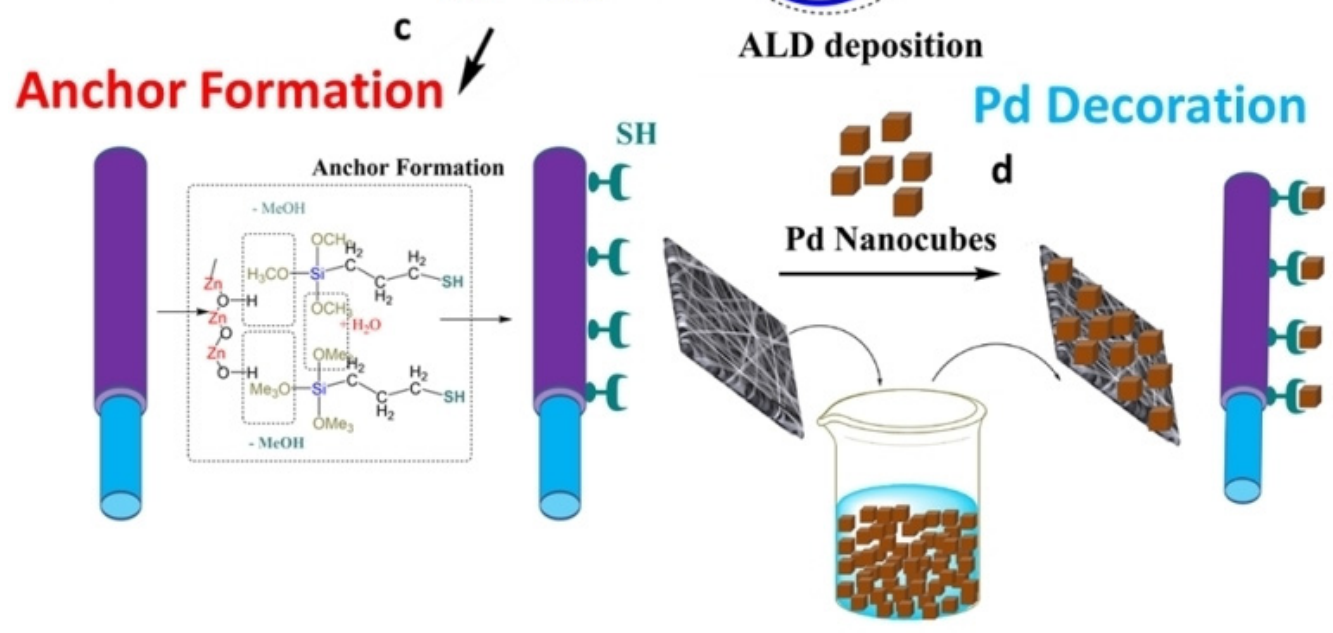

Figure 1. a) Electrospinning of the PAN-NF b) ALD of $\mathrm{ZnO}$ onto electrospun PAN-NF (ZnO@PANNF) c) anchor formation by MPTMS (MPTMS@ZnO@PAN-NF) d) Pd NC surface decoration (Pd@MPTMS@ZnO@PAN-NF). affinity for $\mathrm{ZnO}$ layers and easy to handle features, in the present study, electrospun PAN nanofibers (PAN-NF) were utilized as a suitable nanofibrous template for the ALD of $\mathrm{ZnO}$ nanolayers. ${ }^{3}$ Using this perspective, electrospun PAN-NF was coated with a conformal $\mathrm{ZnO}$ shell layer via ALD. $\mathrm{ZnO}$ is a metal oxide which its surface can be functionalized or decorated by metal nanoparticles for catalysis, sensing and/or optical applications. ${ }^{[7-10]}$ The ALD-coated $\mathrm{ZnO}$ surface features abundance of hydroxyl $(-\mathrm{OH})$ groups left over from the $\mathrm{H}_{2} \mathrm{O}$ halfcycle of the ALD process. ${ }^{[9]}$ Once the polymeric nanofibrous surface is completely covered with metal oxide layer, various kind of surface modification reactions such as anchoring, organic ligand attachment, particle decoration may be possible via sol-gel reactions, physical and/or chemical attachment. ${ }^{[11-12]}$

Nanoparticle decoration onto the electrospun nanofibers provide a vast gallery for the examples of different methods to obtain novel materials for a wide range of applications. ${ }^{[13-14]}$ Combination and uniqueness of the ALD method ${ }^{[15-17]}$ together with electrospinning provided the fabrication of remarkably crystalline and thickness controlled nanoscale metal oxide layers on electrospun polymeric nanofibers. Additionally, metal oxide surfaces with - $\mathrm{OH}$ abundant character can be transformed into active anchor sites by alkoxysilane coupling agents. ${ }^{[18-20]}$ After catalyzed sol-gel reactions to sensitize the metal oxide nanolayer for selective nanoparticle decoration, obtained surfaces become ready for nanoparticle decoration. Some theoretical concepts such as hard-soft acid base concept or affinity increased surfaces is known. ${ }^{[21]}$ From this viewpoint, $\mathrm{PAN} / \mathrm{ZnO}$ core/shell nanofibrous templates were produced with electrospinning and ALD (Figure 1b). This flexible template was further modified with alkoxy alkylthiol groups (MPTMS) for the selective anisotropic Pd NC decoration on the fiber surface. ${ }^{[22-23]}$ Thiol group on the surface resulted in an effective affinity towards the deposition of Pd NC obtained by ligand and facet controlled thermal decomposition technique. Cubic shape provides advantages for a better surface attachment from geometrical perspective. It also provides higher surface areas for complexation, higher yield for TNT degradation, enhanced stability after the catalysis reactions. Additionally, it may increase the local concentration of the $\mathrm{Pd} N C$ on the composite nanofiber. Therefore, $\mathrm{ZnO}$ is vitally important since it provides a solid platform from deposition and efficiency of the Pd NC perspective. Finally, our Pd NC decorated PAN/ZnO core/shell nanofibrous hybrid structure can be utilized for TNT degradation from aqueous solutions by reducing the nitro groups available on the aromatic molecule. ${ }^{[24-28]}$ 


\section{Results and Discussion}

\section{Pd nanocubes (Pd NC) synthesis}

Shape, morphology, size, surface functionality, and other external features regulate the oxidation/reduction strength and character of Pd nanoparticles obtained via reduction, electrochemical and gas phase techniques. ${ }^{[29-30]}$ For the facet controlled synthesis of the $\mathrm{Pd}$ nanocubes ( $\mathrm{Pd} \mathrm{NC}$ ), $\mathrm{Na}_{4} \mathrm{PdCl}_{2} \cdot \mathrm{xH}_{2} \mathrm{O}$ precursor was treated with $\mathrm{PVP}$, ascorbic acid, and $\mathrm{KBr}$ was utilized for the anisotropy control. Thermal decomposition method was employed and conducted at $80^{\circ} \mathrm{C}$ for the synthesis of the $\mathrm{Pd} \mathrm{NC}$ as we reported previously. ${ }^{[31]}$ It is well known that halogen anions $\left(\mathrm{I}^{-}, \mathrm{Br}^{-}, \mathrm{Cl}^{-}\right)$with surface directing agents, control and govern the nuclei formation, growth and facet control of the monodispersed, anisotropic Pd NC. ${ }^{[32-37]}$ Since PVP favors the formation of nanocubes due to the capping on to facets of the growing nanoparticle, Pd nanostructures are synthesized with controlled size and facets. ${ }^{[35}$ Fig. 2 shows the representative TEM and HR-TEM images of the Pd NC with an average size of $\sim 13 \mathrm{~nm}$ (Figure S1). The TEM image (Figure 2a) shows that Pd NC have well-defined cubic shapes with a narrow size distribution. In the HR-TEM image of a single Pd NC (Figure $2 b$ ), lattice fringes are clearly visible where the interatomic distance of $\sim 0.225 \mathrm{~nm}$ is measured, corresponding to the [111] facet. Additionally, single phase $\mathrm{Pd}$ NC can also be detected from the appearance of continuous lattice fringes oriented in the same direction across the cube structure of the Pd NC.

\section{ALD of ZnO nanolayer and surface modification}

For $\mathrm{ZnO}$ deposition, free-standing nanoweb of electrospun PAN nanofibers (PAN-NF) (Figure 3a) was placed into the ALD chamber. After the $\mathrm{ZnO}$ of ALD process, in order to analyze the conformal $\mathrm{ZnO}$ coatings on the electrospun PAN-NF, SEM images (Figure 3b), fiber diameters (Figure S2) and EDX

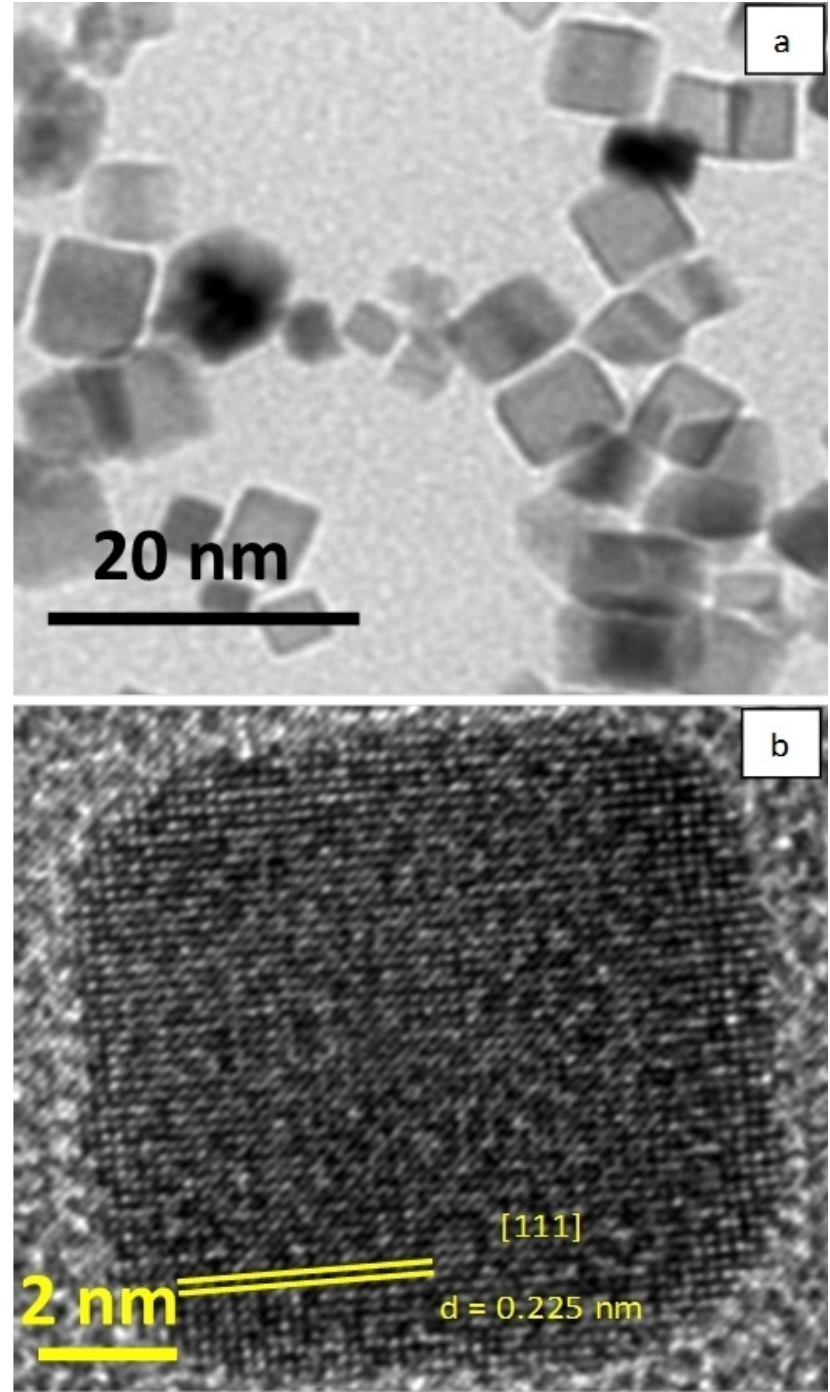

Figure 2. a) Representative TEM image of Pd nanocubes b) HR-TEM image of a single Pd nanocube with its [111] fringe.
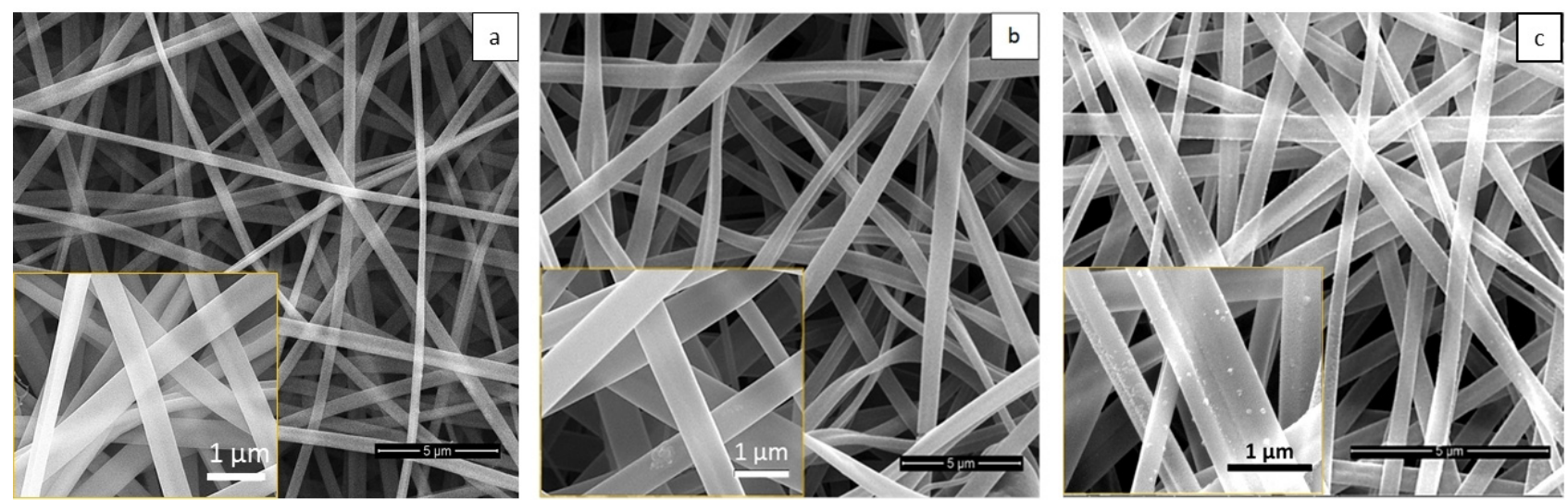

Figure 3. The representative SEM images of a) PAN-NF b) ZnO@PAN-NF c) Pd@MPTMS@ZnO@PAN-NF. High magnification of SEM images are given as inset. 
comparison for PAN-NF and ZnO@PAN-NF data (Figure S3) were investigated. It was observed that, ALD of $\mathrm{ZnO}$ (Figure $3 \mathrm{~b}$ ) and Pd NC decoration (Pd@MPTMS@ZnO@PAN-NF) (Figure 3c) did not change the fibrous morphology of the PAN-NF. Electrospinning of PAN-NF resulted in $550 \pm 200 \mathrm{~nm}$ fiber diameter which increased to $600 \pm 180 \mathrm{~nm}$ after the ALD of ZnO nanolayer (ZnO@PAN-NF) (Figure S2). ZnO@PAN-NF and Pd NC decorated nanofibers (Pd@MPTMS@ZnO@PAN-NF) were further analyzed by EDX mapping (Figure 4), XRD (Figure 5) and XPS
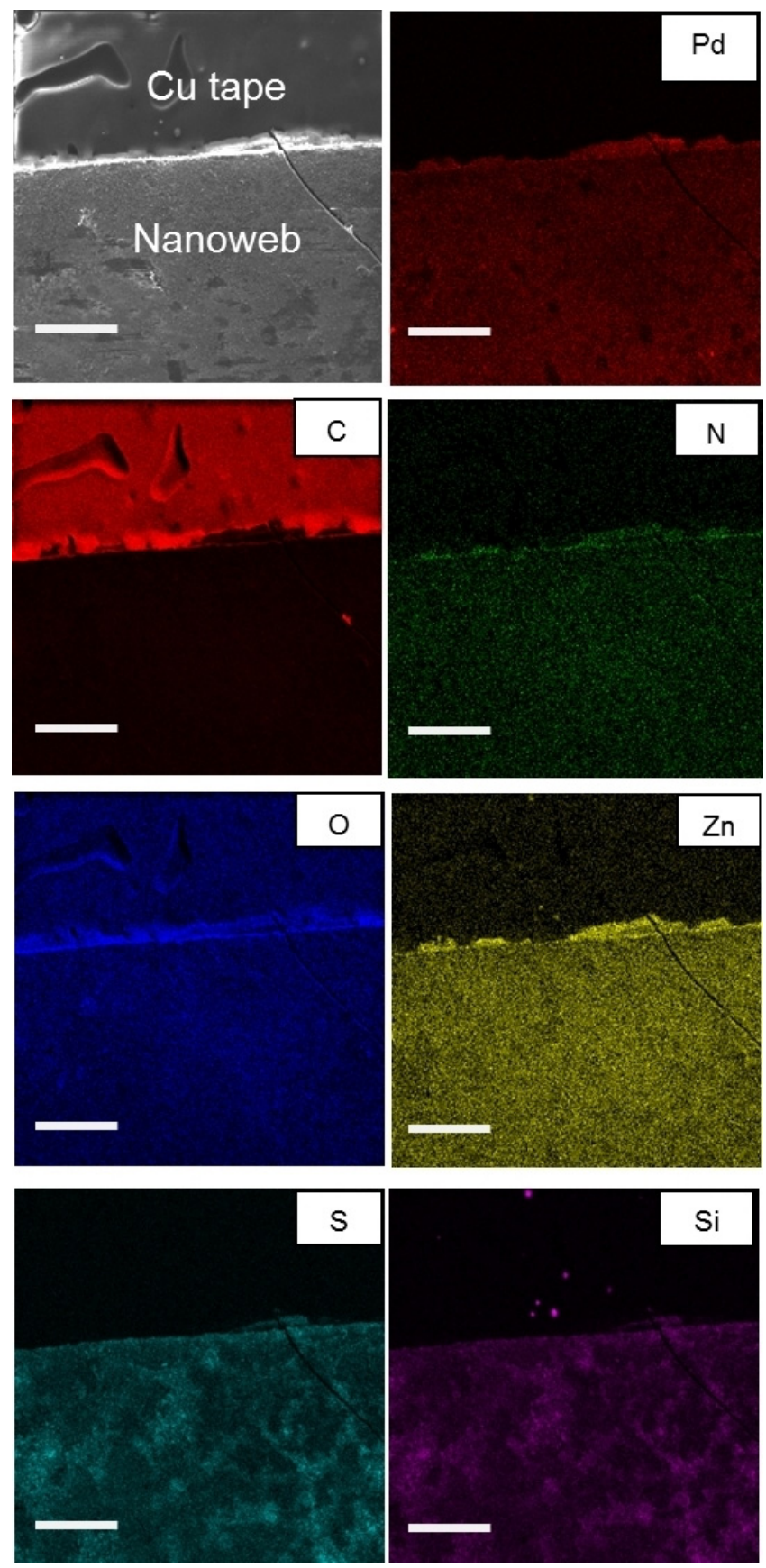

Figure 4. SEM-EDX atomic mapping of Pd@MPTMS@ZnO@PAN-NF nanoweb (scale bar: $50 \mu \mathrm{m}$ ).

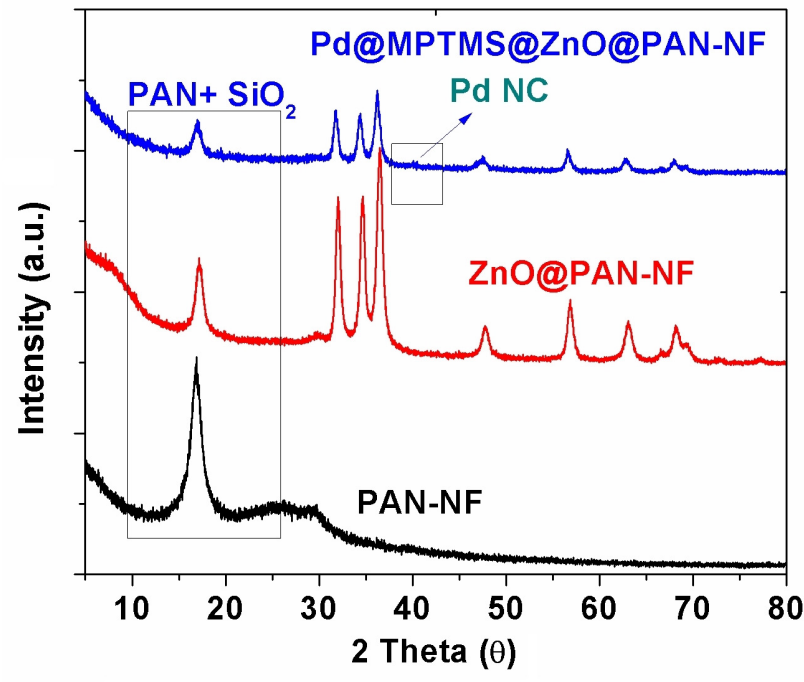

Figure 5. XRD patterns of PAN-NF, ZnO@PAN-NF and Pd@MPTMS@ZnO@PAN-NF samples.

techniques (Figure 6). SEM-EDX mapping of the Pd@MPTMS@ZnO@PAN-NF (Figure 4) showed each available atomic composition. Data of SEM-EDX mapping revealed that $\mathrm{Pd}, \mathrm{Zn}, \mathrm{O}, \mathrm{N}, \mathrm{Si}, \mathrm{C}$ and $\mathrm{S}$ atoms were present in the Pd@MPTMS@ZnO@PAN-NF sample. Results showed that Pd NC are homogeneously distributed on the flexible web of Pd@MPTMS@ZnO@PAN-NF (Figure 4).

XRD study reveals the high crystalline nature of the deposited ZnO coating onto PAN-NF (Figure 5). High crystallinity of the fabricated $\mathrm{ZnO}$ nanolayer is highly desired which may increase the advantages of surface properties. EDX analysis on the deposited surfaces was employed (Figure S3) to show the purity of the ZnO layer on the PAN-NF. Same observations were done by XRD diffraction pattern comparison and XPS (Figure 6a-c) analysis of the Pd@MPTMS@ZnO@PAN-NF before and after the catalytic degradation experiments. When XRD patterns of PAN-NF, Zn@PAN-NF and Pd@MPTMS@ZnO@PANNF nanocomposite are compared (Figure 5), PAN structure shows its usual $2 \theta=\sim 17$ position and additionally most common ZnO peaks for ZnO@PAN-NF appear at [100], [002] and [101] for hexagonal wurtzite structure of ZnO (JCPDS:361451). MPTMS anchoring did not change XRD patterns but a weak diffraction line at $2 \theta=40.15^{\circ}$, which corresponds to the metallic Pd (111) with face-centered cubic structure was detected for the Pd@MPTMS@ZnO@PAN-NF sample.

Literature revealed that reflection peaks for $\mathrm{Pd}$ can be observed at $40.1^{\circ}, 46.7^{\circ}, 67.8^{\circ}, 82.1^{\circ}$, and $86.4^{\circ}$, which correspond to the [111], [200], [220], [311] and [222] planes of Pd (JCPDS, card no. 46-1043), respectively. ${ }^{32}$ Therefore, it is possible to conclude that $\mathrm{Pd} \mathrm{NC}$ are available on the nanocomposite structure after the decoration procedure. As discussed below, XPS (Figure 6) and TEM (Figure 7) results for Pd@MPTMS@ZnO@PAN-NF revealed the presence of Pd NC on the nanocomposite structure. 

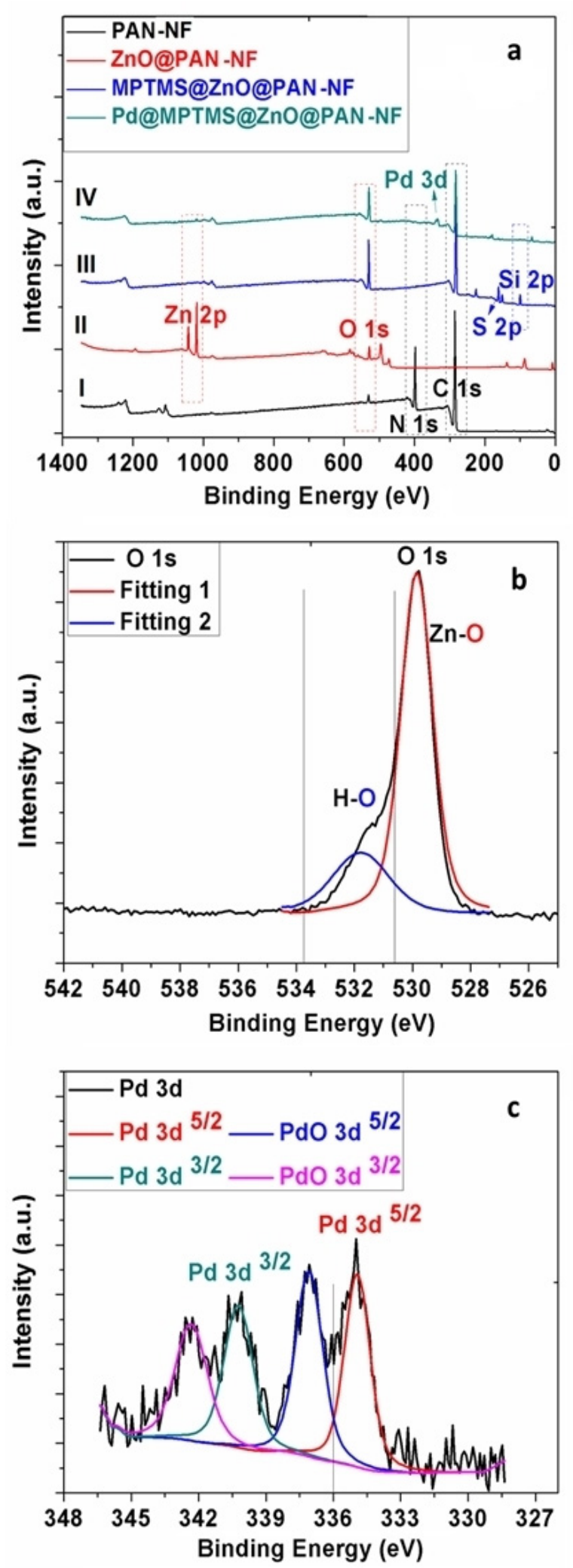

Figure 6. a

XPS survey of nanofibrous samples; I) PAN-NF, II) ZnO@PAN-NF, III) MPTMS@ZnO@PAN-NF and IV)Pd@MPTMS@ZnO@PAN-NF b) High resolution O1s XPS spectrum for the ZnO@PAN-NF c) High resolution Pd 3d XPS spectrum for Pd@MPTMS@ZnO@PAN-NF.
The fiber thickness increase in ZnO@PAN-NF is compatible with the measured thickness $(\sim 40 \mathrm{~nm})$ of $\mathrm{ZnO}$ coating obtained by TEM analysis (Figure 7a-d).This increase also confirmed the high-degree of $\mathrm{ZnO}$ conformality. Conformal $\mathrm{ZnO}$ coating on ZnO@PAN-NF provides a suitable platform for the further alkoxysilane modification. Since diethylzinc $\left(\mathrm{Zn}(\mathrm{Et})_{2}\right)$ together with $\mathrm{H}_{2} \mathrm{O}$ were employed as reactants for $\mathrm{ZnO}-\mathrm{ALD}$, thickness control of the deposited layers with enhanced crystallinity is a key point while water vapor can provide -OH groups (Figure $6 \mathrm{~b}$ ) on the surface having the advantage of reacting with alkoxy groups in modification and Pd NC decoration process (Figure 1c-d). Analyzed SEM pictures after the deposition showed that nanofiber structure of the electrospun PAN-NF was preserved and there is no visible morphological change in the overview except an observable increase in fiber diameter from TEM analysis (Figure 7a-d). Organic PAN part is observed in the core as slightly brighter region, however, local inhomogenities can also be detected from measurements. It is evident that deposited $\mathrm{ZnO}$ shell layer has a rather uniform thickness about the targeted thickness of $40 \mathrm{~nm}$. ALD deposition caused slightly rougher morphology due to the grainy growth nature of polycrystalline ZnO. HR-TEM images of this core/shell morphology revealed that the thickness of $\mathrm{ZnO}$ shell layer was quite uniform throughout the nanofibrous polymeric PAN template (Figure 7a-b).

Most salient advantage of the $\mathrm{ZnO}$ deposition is its surface characteristics serving as a perfect platform for a designed and stable Pd NC decoration. Ex-situ decoration methods suffer from low surface area for catalysis, low stability, low catalytic yield and additionally embedded Pd nanostructures show low catalytic efficiency due to the veiled particle surface.Hence, $\mathrm{ZnO}$ deposition and its further modification with sol-gel chemistry remarkably reduces these drawbacks. Crystalline $\mathrm{ZnO}$ layer provides a better, flexible (Figure S4) interface between $\mathrm{ZnO}$ and Pd NC due to the alkyl thiol anchors which can not be obtained by embedded Pd NC/PAN NF. This can also be detected by the TEM investigation and EDX analysis (Figure S4). After anchoring, increased surface area of the Pd NC, geometrical positioning on fiber, efficient catalytic yield, remarkable stability were obtained for the repeatable catalytic reduction of 2,4,6-trinitrotoluene (TNT) in aqueous medium. As blank investigations, Pd decoration efficiency was detected on PANNF, ZnO@PAN-NF with EDX atomic mapping and compared with MPTMS modified structure with XPS (Figure S5).

For an efficient catalysis, $\mathrm{ZnO}$ surface should be modified with an alkyl thiol alkoxysilane coupling agent for the anchor points on this metal oxide layer. Basic sol-gel chemistry with hydrolysis and condensation reactions on the $\mathrm{ZnO}$ nanolayer resulted with -SH decorated surfaces as revealed by XPS (Figure 6a, blue). After the anchor formation, Pd NC were deposited onto this layer (MPTMS@ZnO@PAN-NF) which was analyzed and confirmed by XPS (Figure 6a-c) and HR-TEM (Figure $7 d$ inset). In Figure $7 c$ and in Figure $7 d$, STEM and HRTEM images reveal the Pd NC on the core/shell (ZnO@PAN-NF) structures with MPTMS and a single Pd nanocube was clearly observed with its fringes. It is well known that conformal and layer-by-layer deposition of metal oxides onto complex three- 

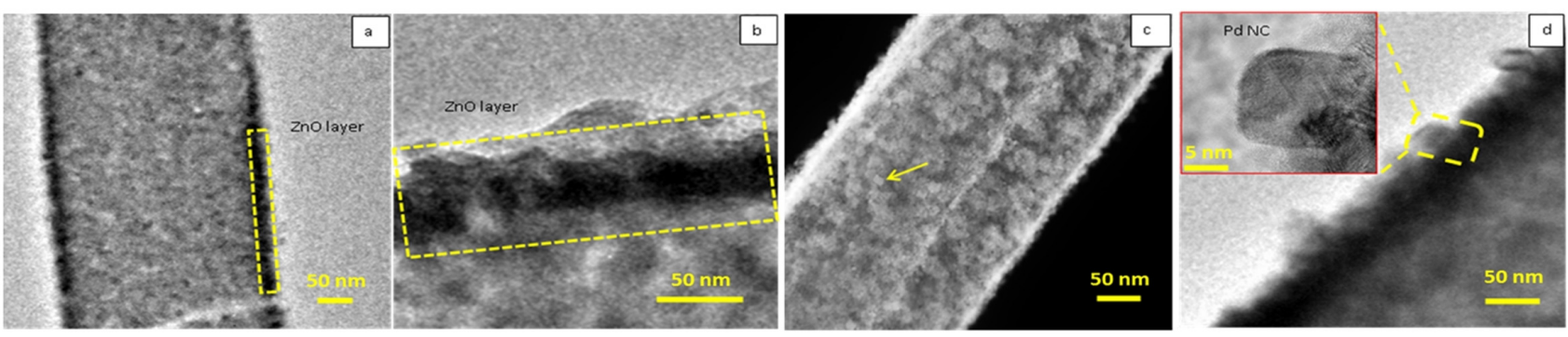

Figure 7. a) Representative TEM image of the ZnO@PAN-NF b) Enlarged view for the ZnO nanolayer c) STEM image for Pd@MPTMS@ZnO@PAN-NF d) Enlarged image for the edge of the Pd@MPTMS@ZnO@PAN-NF HR-TEM image of the Pd NC as inset.

dimensional surfaces (such as electrospun nanofibers) is absolutely challenging. ${ }^{[38]}$ Owing to the optimized ALD growth conditions, homogeneous $\mathrm{Pd} \mathrm{NC}$ decoration onto the -SH anchored ZnO shell layer (Pd@MPTMS@ZnO@PAN) was detected by the HAADF-STEM-EDS mapping which is presented in Figure 8. HDAAF-STEM has provided a great clarity for the gradients of each atom available on the final nanocomposite system. As presented, $\mathrm{C}$ and $\mathrm{N}$ mapping covers the main inner core and $\mathrm{Zn}$ in conjunction with $\mathrm{O}$, which is also abundant is seen as expected. $\mathrm{Si}, \mathrm{S}$, and $\mathrm{Pd}$ mapping reveals that they were positioned on the nanofiber surface. Oxygen and Zinc range is relatively denser due to the radial coverage of the PAN-NF surface. Small grains of the $\mathrm{ZnO}$ on the PAN nanofiber is also noticeable. The Pd NC were observed and Pd NC were seen on the anchor points interacting with the -SH groups. $\mathrm{Pd} \mathrm{NC}$ decoration onto MPTMS@ZnO@PAN-NF reveals a distinctive character and it is possible to observe the surface chemical composition and bonding states of the coated and pristine PAN-NF by XPS (Figure 6a-c).

According to the measured XPS peaks (Figure 6), electrospun PAN-NF showed only 2 dominant peaks as $C 1 \mathrm{~s}$ and $\mathrm{N} 1 \mathrm{~s}$ at $237 \mathrm{eV}$ and $398 \mathrm{eV}$, respectively (Figure 6a-l). PAN is composed of $\mathrm{C}$ and $\mathrm{N}$, however a small $01 \mathrm{~s}$ peak is also observable possibly arising due to atmospheric contamination. XPS survey analysis comparison of the PAN-NF and ZnO@PAN-NF (Figure 6a-II) very clearly shows that PAN surface contains $\mathrm{ZnO}$

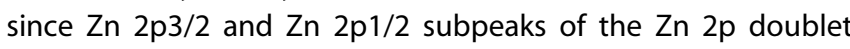
are located at 1020.90 and $1044.02 \mathrm{eV}$, respectively (Figure S6). In addition, - $\mathrm{OH}$ groups positioned on the $\mathrm{ZnO}$ surface can be detected as shown in Figure $6 \mathrm{~b}$. High resolution peaks are presented in Figure S6 for ZnO@PAN-NF and N 1s peak seemed completely veiled due to the relatively thick $\mathrm{ZnO}$ shell layer. In the corresponding $395-400 \mathrm{eV} \mathrm{N} 1 \mathrm{~s}$ region, there is no peak observed (Figure S6b). C 1s peak is composed of two different peaks at $288.4 \mathrm{eV}$ and $284.9 \mathrm{eV}$ corresponding to the artifact $\mathrm{C}$ $1 \mathrm{~s}$ or adsorbed species. In the enlarged O1s XPS peak for $\mathrm{ZnO}$ layer, peak deconvolution resulted in $-\mathrm{OH}$ groups which can be used for the MPTMS functionalization.

Since $-\mathrm{OH}$ peaks are capable of reacting with the alkoxy groups and propagating the controllable hydrolysis and condensation reactions, - $\mathrm{SH}$ anchor positions for $\mathrm{Pd} \mathrm{NC}$ was formed. ${ }^{[39-41]} \mathrm{Pd}$ NC decoration was simply employed with EtOH solutions and dipping process (Figure 1c-d). After functionaliza-
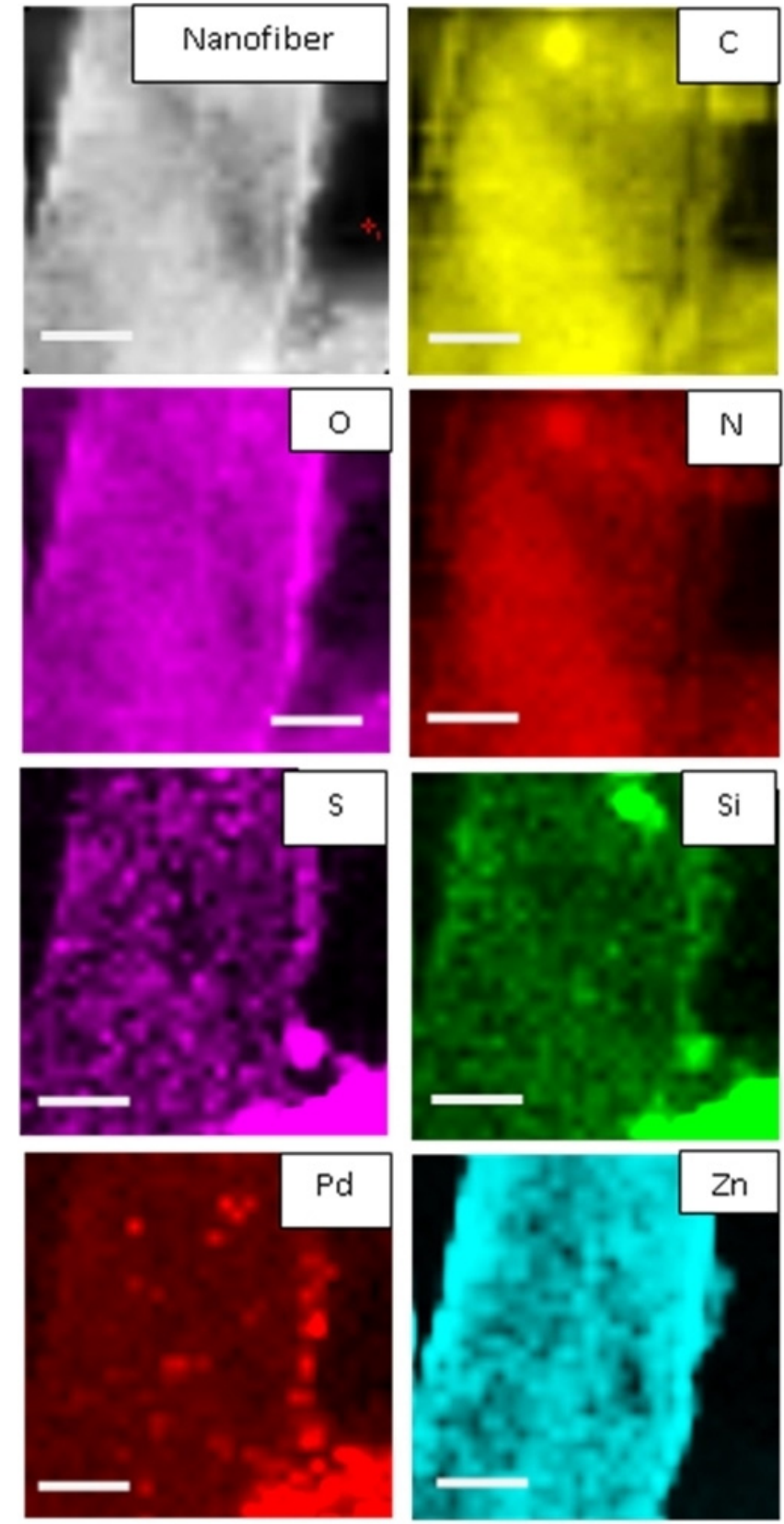

Figure 8. HAADF-STEM-EDX mapping images for the Pd@MPTMS@ZnO@PAN-NF (scale bar: $200 \mathrm{~nm}$ ). 
tion, XPS analysis was conducted to examine the -SH anchor points and bonding states of the thiol group. When XPS is used before and after the MPTMS functionalization, high-resolution core level XPS peaks for $S 2 p$ and Si $2 p$ have revealed their characteristic peaks (Figure S7). Intensity of the Zn $2 p$ peak reduced due to the silica network on the surface, however, it is still detectable. Additionally, after MPTMS functionalization, XPS spectra of the ZnO coating is shown in Figure 6a-III. XPS S2p core level spectrum of MPTMS functionalized $\mathrm{ZnO}$ layer exhibited one double peak which consists of a spin-orbitsplitting doublet with the $S(2 p 3 / 2)$ and $S(2 p 1 / 2)$ binding energy positions of $163.8 \mathrm{eV}$ and $165 \mathrm{eV}$, respectively (Figure S7b). Positions of these peaks are consistent with the binding energy of unbound thiol (-SH) and literature values ${ }^{[42-46]}$ Therefore, the spin-orbit coupled doublet for the $S(2 \mathrm{p} 3 / 2)(2 \mathrm{p} 3 / 2,2 \mathrm{p} 1 / 2)$, which is extracted as 2:1 and the energy split was exactly $1.2 \mathrm{eV}$. These results indicated that the MPTMS molecules were efficiently attached onto the ALD-ZnO layer (MPTMS@ZnO@PAN). In addition to that, core level, XPS Si $2 p$ showed a peak at $102.5 \mathrm{eV}$ which can be associated with and $\mathrm{S}(2 \mathrm{p} 1 / 2)$ binding energy positions. Furthermore, peaks at 163.8 and $165 \mathrm{eV}$ can be attributed to unbound thiol (-SH) of the MPTMS molecules on the $\mathrm{ZnO}$ surface. Curve fitting procedure resulted in relative ratios for each spin-orbit coupled pair the $\mathrm{Si}$ or thin polymerized alkoxy silane layer. From these investigations we can conclude that there was sufficient and ready mercapto (thiol) anchor Pd NC deposition on ZnO coatings. XPS (Figure 6a-IV) showed the interaction between $\mathrm{S}$ atoms of MPTMS and the Pd NC during the formation of thiol-stabilized Pd NC. XPS spectra (Figure 6a, Figure S8) of the Pd, Si, Zn, and $\mathrm{S}$ showed that all anticipated groups are detectable separately. For Pd NC double peaks with binding energies of 334.8 and $339.9 \mathrm{eV}$, corresponding to $\mathrm{Pd} 3 \mathrm{~d}^{5 / 2}$ and $\mathrm{Pd} 3 \mathrm{~d}^{3 / 2}$, respectively, were observed. In addition, oxidized $\mathrm{PdO}$ peaks were also detected at 337.3 and $342.4 \mathrm{eV}$, the respectively. According to the XPS analysis, \%39 typical valence state peaks of PdO and are found to be completely consistent with the literature values. $^{[47-50]}$ After anchoring, for $\mathrm{Si} 2 \mathrm{p}$, binding energy of $102.5 \mathrm{eV}$ is observed which is a sign that there is no shifting for the Si peak. After Pd NC decoration, Zn $2 p$ peaks have been observed at 1044.2 and $1021.1 \mathrm{eV}$, respectively for $\mathrm{Zn} 2 \mathrm{p} 1 / 2$ and $\mathrm{Zn} 2 \mathrm{p} 3 / 2$. XPS peak for $\mathrm{S}$ was observed in two positions. First peak appears at $162.7 \mathrm{eV}$ which is typical formetal-bound S2p value and $168.1 \mathrm{eV}$ for the oxidated sulphur (SOx) species (Figure S7). ${ }^{[51-55]}$ Oxidation possibly occurs due to the applied drying procedure. When compared, intensity of the metalbound S2p peak was remarkably high.

\section{Catalytic Reduction of TNT}

In order to evaluate efficiency of the Pd@MPTMS@ZnO@PAN$\mathrm{NF}$, catalytic reduction experiments for the TNT and para-nitro phenol (PNP) were conducted. According to the TEM and STEM images, Pd NC have a monodispersed distribution and owing to our multi-step fabrication technique, surface of the Pd NC maintained their clean and large surface area and stability. When $\mathrm{Pd}$ nanostructures are covered with ligands or em- bedded in polymers surface pollution or decrease in catalytic activity is observed due to reducement in the coordination sites of the metal nanoparticles. ${ }^{[56-57]}$ For the catalytic degradation of TNT,active sites of the Pd NC for nitro groups together with 5 ring intermediate is essential. ${ }^{[58]}$ UV-visible absorption technique can be used to determine the concentration of the TNT reduced by the Pd@MPTMS@ZnO@PAN-NF. During reduction process obtained by nanocomposite structure, $\mathrm{BH}_{4}^{-}$and TNT should be adsorbed onto the Pd NC forming the active intermediate hydride complex to transfer it onto the TNT. The reduction of the nitro group generally does not proceed in the absence of the catalyst because the kinetic barrier is high and should be overcome. Adsorption steps are reversible and can be analyzed by basic adsorption models. Transferred electrons cause an energy barrier according to the Langmuir-Hinselwood theory ${ }^{[59]}$ and intensity at the corresponding region decreases. When reduced molecules on metal particle are formed, they leave the surface and next cycle begins. Therefore, in a typical $1 \mathrm{mM}$ TNT reduction experiment, stock solution of the TNT solution was prepared as $4 \mathrm{~mL}$ and certain concentration of the freshly prepared $3 \mathrm{~mL} \mathrm{NaBH}$ solution was added into the TNT solution. Spectral variations in the solution were monitored at a certain time intervals by employing a UV-vis spectrophotometer under constant $100 \mathrm{rpm}$ stirring. Variations in the TNT concentration can be calculated from the UV-Vis absorption spectrum which will be used to determine the kinetic data. Blank experiments were also conducted with only PAN-NF and ZnO@PAN-NF. Additionally to show the inefficient Pd NC decoration onto PAN-NF and ZnO@PAN-NF, blank experiments with Pd NC decoration were conducted (Figure S9). According to the results,Pd@MPTMS@ZnO@PAN-NF containing 1 mM TNT solution reduced the TNT within 20 seconds while pristine mixture without any fiber catalyst required 25 minutes to reduce \%75 of the TNT (Figure 9). Therefore, one cannot mention about the full transformation of the TNT without catalyst probably due to the ionic barrier. PAN-NF and ZnO@PAN-NF needed 21 and 20 minutes respectively to degrade the TNT as control experiments (Figure S9a-b). Pd NC decoration efficiency was also detected on PAN-NF (PdNC@PAN-NF) and ZnO@PAN-NF (PdNC@ZnO@PAN-NF) and resulted more than 20 minutes for each case (Figure S9c). The photos of the reduced TNT solutions were presented in Figure $9 a$ and $9 b$ (inset). Since Pd NC positioned on the surface of the MPTMS@ZnO@PAN-NF via mercapto anchor groups and nanofiber template affer remarkably high surface area, Pd NC provided an extreme enhancement in catalytic efficiency for TNT reduction. There are 3 regions observed within the UV-Vis spectrum during the TNT reduction. According to the pure TNT UV-Vis spectra, there is no remarkable absorption after $280 \mathrm{~nm} \cdot{ }^{[60-63]}$ When reductant $\mathrm{BH}_{4}^{-}$is added, complex products show strong peaks at different positions of the spectra. First one ranges between $327 \mathrm{~nm}$ and $570 \mathrm{~nm}$ having the $408 \mathrm{~nm}$ as peak point. Second peak became visible after 20 second and has the $293 \mathrm{~nm}$ value. Additionally complex products show strong peaks at with $232 \mathrm{~nm}$ peak. To evaluate the efficiency, we conducted the repetitive reduction experiments for TNT in addition to the p-nitrophenol (PNP) reduction experiments 

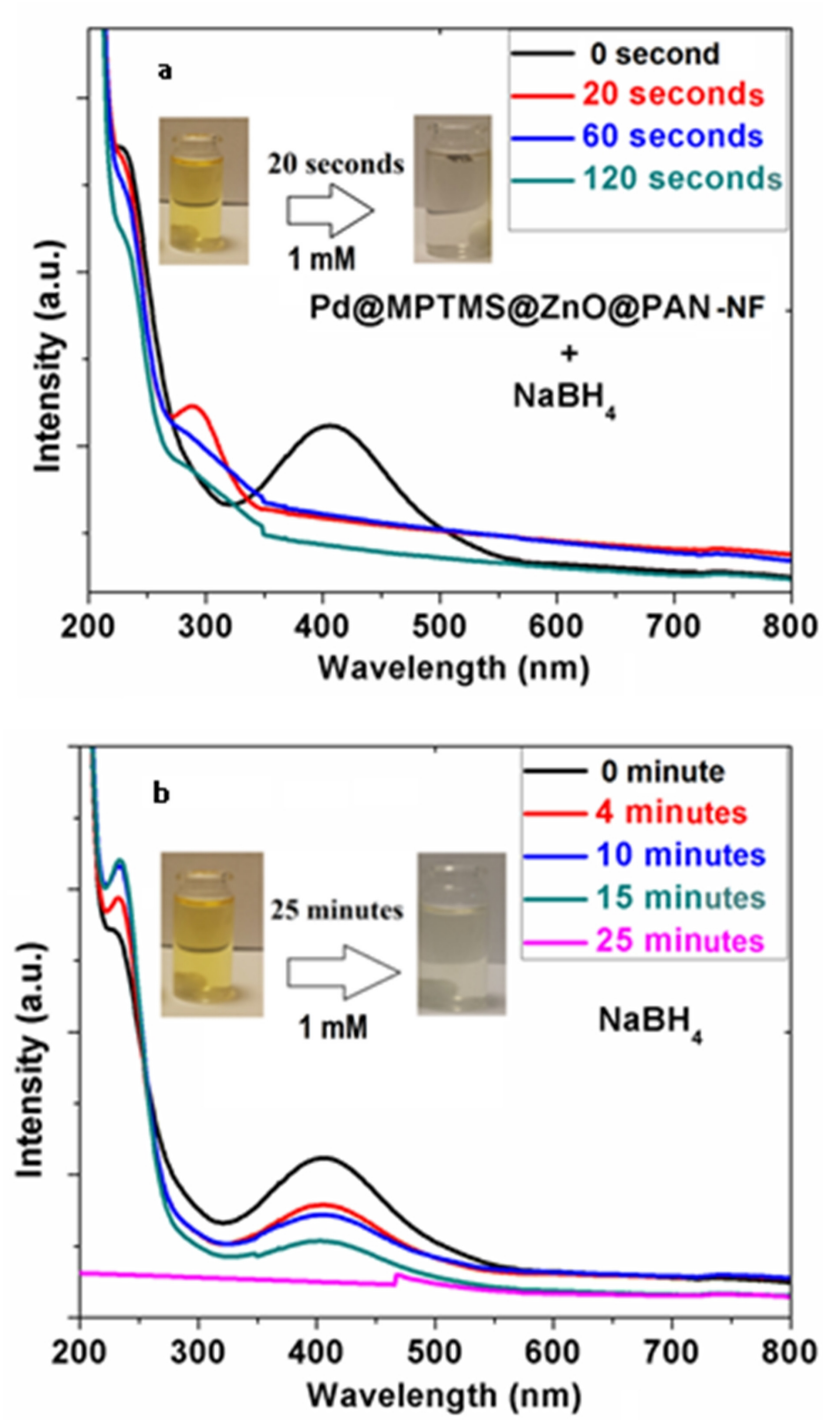

Figure 9. UV-Vis observation and comparison of the catalytic efficiency for the a)Pd@MPTMS@ZnO@PAN and b) control experiment without any fiber with their corresponding visual observation after full catalytic reduction (insets).

(Figure S10). Upon washing with acetone and ethanol, followed by drying at $80^{\circ} \mathrm{C}$, the Pd@MPTMS@ZnO@PAN-NF was used for subsequent iterative experiments. Results clearly showed that, within the same catalytic experiment periods, the conversion was always $\% 100$ and sometimes in the $\% 98-100$ range which fits well with the expected limits for PNP. According to the results,Pd@MPTMS@ZnO@PAN efficiency did not change for six sequential experiments for TNT reduction and four experiments for PNP reduction. This also proves the remarkable stability and reusabe efficiency of our well-designed Pd@MPTMS@ZnO@PAN-NF nanocomposite system. SEM images and EDX analysis after the catalytic experiments (Figure S11) together with XRD and XPS investigations (Table S1) revealed that $\mathrm{Pd}$ nanocubes are still on the nanofibrous web. XPS data also proved that there is no remarkable decrase in Pd concentration after the catalytic reduction. XRD pattern (Fig- ure S12a) of the Pd@MPTMS@ZnO@PAN-NF after the catalytic degradation experiment showed that the $\mathrm{Pd} N C$ peak is detected at $2 \theta=40.15$. Additionally, HR-XPS results (Figure $\mathrm{S} 12 \mathrm{~b}$ ) of the Pd NC showed double peaks with the binding energies of 334.2-334.9 range and 339.8-340.2 eV range, corresponding to $\mathrm{Pd} 3 \mathrm{~d}^{5 / 2}$ and $\mathrm{Pd} 3 \mathrm{~d}^{3 / 2}$, respectively. Additionally, oxidized $\mathrm{PdO}$ peaks were also detected at 337.3-337.6 range and $342.4-342.6 \mathrm{eV}$ range, respectively. Latter XPS values are expected valence state peaks of $\mathrm{PdO}$ and consistent with the literature values. ${ }^{[64]}$ After the catalytic experiments, even though the amount of the total Pd is relatively decreased, oxidated $\mathrm{Pd}$ amount seems relatively enhanced. Quantitative results revealed that \% $0.96 \mathrm{Pd}$ is available before the catalytic degradation experiment which decreased to $\% 0.79$ after the degradation (Figure S12c). This is relatively low and roughly $\% 10$ decrease from the total amount which was detected showing the remarkable stability of the Pd NC in this nanocomposite system. Catalytic reduction experiments of the PNP also showed similar results. PNP reduction reaction was completed within 25 seconds and the yellow solution became colorless indicating the full transformation of the PNP into its corresponding product. Previous catalytic studies showed that when Pd nanoparticles were used in different forms, architectures or supporting materials; some literature results showed that 2 fold or 4 fold or even 12 fold increase/variation in catalytic activity can be observed. ${ }^{[65]}$ This is probably due to the reduced surface coverage and surface activity and organic ligand adsorption preventing active complex formation on the $\mathrm{Pd}$ surface. But in this study we have shown that with better attachment of Pd NC on ZnO nanolayer, possibly due to their cubic shape, remarkably stable interaction was provided. This also enhanced efficiency, repeatability, stability after the catalytic reactions and minimize the Pd NC leaching as revealed by our analyses.

\section{Conclusions}

We have shown that fabricated electrospun PAN nanofibers can be conformally coated with precisely controlled $\mathrm{ZnO}$ nanolayers by ALD technique. These flexible templates can be decorated by $\mathrm{Pd}$ NC for the catalytic reduction of TNT. Since ex-situ deposited Pd nanostructures suffer from the low catalytic activity, cubic nanoparticles enhanced interface properties, attachment quality, catalysis yield and stability after the catalysis reactions. Due to the increased surface interaction, better geometrical positioning on nanofiber for a better intermediate complex formation and stability via soft-soft interaction, flexible polymeric electrospun nanowebs were produced for the enhanced and repeatable catalytic reduction of TNT. Obtained nanocomposite material provided 20 second catalytic reduction time period for $1 \mathrm{mM}$ TNT which is remarkably fast when compared to same experiment conducted without or inefficiently Pd NC decorated nanofibrous materials with PdNC@PAN-NF and PdNC@ZnO@PAN-NF ( 20 minutes). Additionally without the nanofibrous material normal experiment took $\sim 25$ minutes for a complete reduction (Figure 9a-b). XPS and SEM investigations resulted with a 
durable, flexible and efficient catalytic reduction with remarkable efficiency along with reusability.

\section{Acknowledgements}

Prof. Arslan acknowledges TUBITAK BIDEB Fellowships for 2232Post Doctoral programme (project no. 115C095) for financial support. T.U acknowledges partial support of The Turkish Academy of Sciences-Outstanding Young Scientists Award Program (TUBA-GEBIP). The authors acknowledge Dr. F. Topuz for the synthesis of $\mathrm{Pd} \mathrm{NC}$ and thank to M. Güler for TEM investigations.

\section{Conflict of Interest}

The authors declare no conflict of interest.

Keywords: ALD - Catalytic reduction - Electrospinning Nanofibers $\cdot$ Palladium nanocubes $\cdot$ TNT

[1] X. Sun, Y. Wang, Y. Lei, Chem. Soc. Rev. 2015, 44, 8019-8061.

[2] F. Kayaci, C. Ozgit, I. Donmez, N. Biyikli, T. Uyar, ACS Appl. Mater. Interfaces 2012, 11, 6185-6194.

[3] F. Kayaci, S. Vempati, C. Ozgit-Akgun, N. Biyikli, T. Uyar, Appl. Catal. B 2014, 156, 173-183.

[4] C. Akgun, F. Kayaci, S. Vempati, A. Haider, A. Celebioglu, E. Goldenberg, S. Kizir, T. Uyar, N. Biyikli, J. Mater. Chem. C 2015, 3, 5199-5206.

[5] F. Kayaci, C. Ozgit-Akgun, N. Biyikli, T. Uyar, RSC Adv. 2013, 3, 6817.

[6] F. Kayaci, S. Vempati, I. Donmez, N. Biyikli, T. Uyar, Nanoscale 2014, 6, 10224-10234.

[7] W. Lin, Y. Chiu, P. Shao, Y. Hsu, ACS Appl. Mater. Interfaces 2016, 48, 32754-32763.

[8] O. Baffa, D. R. Clarke, Sci. Rep. 2015, 5, 14004-14015.

[9] R. M. Hewlett, M. A. McLachlan, Adv. Mater. 2016, 28, 3893-921.

[10] N. H. Moreira, A. Dominguez, T. Frauenheim, A. L. Rosa, Phys. Chem. Chem. Phys. 2012, 14, 15445-15451.

[11] O. Arslan, L. Belkoura, S. Mathur, J. Mater. Chem. C 2015, 3, 11965-11973.

[12] B. Dinga, T. Ogawa, J. Kima, K. Fujimoto, S. Shiratori, Thin Solid Films 2008, 516, 2495-2501.

[13] C. Zhang, S. Yu, Chem. Soc. Rev. 2014, 43, 4423-4448.

[14] O. Arslan, T. Uyar, Nanoscale 2017, 9, 9606-9614.

[15] M. Leskela, M. Ritala, Angew. Chem. Int. Ed. 2003, 42, 5548-54.

[16] S. M. George, Chem. Rev. 2010, 110, 111-131.

[17] C. Detavernier, J. Dendooven, S. P. Sree, K. F. Ludwig, J. A. Martens, Chem. Soc. Rev. 2011, 40, 5242-53.

[18] M. lijima, M. Tsukada, H. Kamiya, J Colloid Interface Sci. 2007, 305, 315323.

[19] O. Arslan, Z. Aytac, T. Uyar, J. Mater. Chem. C 2017, 5, 1816.

[20] H. Shi, W. Li, L. Sun, Y. Liu, H. Xiao, S. Fu, Chem. Commun. 2011, 47 11921-11923.

[21] J. A. Lemire, J. J. Harrison, R. J. Turner, Nat. Rev. Microbiol. 2013, 11, $371-$ 384.

[22] Y. Sun, M. Chen, S. Zhou, J. Hu, L. Wu, ACS Nano 2015, 12, 12513-12520.

[23] C. Jia, F. Schüth, Phys. Chem. Chem. Phys. 2011, 13, 2457-2487.

[24] R. Zhang, C. L. Sun, Y. J. Lu, W. Chen, Anal. Chem. 2015, 87, 12262-12269.

[25] S. M. El-Sheikh, A. A. Ismail, J. F. Al-Sharab, New J. Chem. 2013, 37, 23992407.

[26] I. Saldan, Y. Semenyuk, I. Marchuk, O. Reshetnyak, J Mater Sci. 2015, 50, 2337-2354.

[27] A. Balanta, C. Godard, C. Claver, Chem. Soc. Rev. 2011, 40, 4973-4985.

[28] R. A. Soomro, O. P. Akyuz, H. Akin, R. Ozturk, Z. H. Ibupoto, RSC Adv. 2016, 6, 44955-44962.
[29] H. You, S. Yang, B. D. H. Yang, Chem. Soc. Rev. 2013, 42, 2880-2904.

[30] S. Yang, C. Shen, Y. Tian, X. Zhang, H. Gao, Nanoscale 2014, 6, 13154

[31] O. Arslan, F. Topuz, H. Eren, N. Yildirim, T. Uyar, New J. of Chem 2017, 41, 4145-4156.

[32] H. K. Kadam, S. G. Tilve, RSC Adv. 2015, 5, 83391-83407.

[33] A. Chen, C. Ostrom, Chem. Rev. 2015, 115, 11999-12044.

[34] T. Teranishi, M. Miyake, Chem. Mater. 1998, 10, 594-600.

[35] Y. Xiong, Y. Xia, Adv. Mater. 2007, 19, 3385-3391.

[36] R. Long, H. Huang, Y. Li, L. Song, Y. Xiong, Adv. Mater. 2015, 27, 70257042.

[37] B. Lim, M. Jiang, J. Tao, P. H. C. Camargo, Y. Zhu, Y. Xia, Adv. Funct. Mater. 2009, 19, 189-200.

[38] L. Chen, L. Bromberg, J. A. Lee, H. Zhang, H. S. Gibson, P. Gibson, J. Walker, P. T. Hammond, T. A. Hatton, G. C. Rutledge, Chem. Mater. 2010, 22, 1429-1436.

[39] R. D. Badley, W. T. Ford, F. J. McEnroe, R. A. Assinks, Langmuir 1990, 6, 792-801.

[40] F. Sinapi, J. Delhalle, Z. Mekhalif, Mater. Sci. Eng. C 2002, 22, 148-154.

[41] E. Casero, M. D. Petit-Domínguez, M. Barbadillo, F. Pariente, L. Vázquez, E. Lorenzo, Analyst 2011, 136, 340-347.

[42] A. Guarnizo, I. Angurell, M. D. Rossell, J. Llorca, G. Muller, M. Seco, O. Rossell, RSC Adv. 2015, 5, 91340-91348.

[43] L. Forget, J. Delhalle, Z. Mekhalif, Appl. Surf. Sci. 2003, 212, 464-471.

[44] J. Singh, J. E. Whitten, J. Phys. Chem. C 2008, 112, 19088-19096.

[45] S. Ungureanu, H. Deleuze, C. Sanchez, M. I. Popa, R. Backov, Chem. Mater. 2008, 20, 6494-6500.

[46] V. Romero, I. Costas-Mora, I. Lavilla, C. Bendicho, Nanoscale 2015, 7, 1994-2002.

[47] F. G. Rivera, I. Angurell, M. D. Rossell, R. Erni, J. Llorca, N. J. Divins, G. Muller, M. Seco, O. Rossell, Chem. - Eur. J. 2013, 19, 11963-11974.

[48] Z. D. Pozun, S. E. Rodenbusch, E. Keller, K. Tran, W. Tang, K. J. Stevenson, G. A. Henkelman, J. Phys. Chem. C 2013, 117, 7598-7604.

[49] K. Kim, K. L. Kim, K. S. Shin, J. Phys. Chem. C 2011, 115, 14844-14851.

[50] R. Bhandari, M. R. Knecht, ACS Catal. 2011, 1, 89-98.

[51] J. C. Love, L. A. Estroff, J. K. Kriebel, R. G. Nuzzo, G. M. Whitesides, Chem. Rev. 2005, 105, 1103-69.

[52] P. Carro, G. Corthey, A. A. Rubert, G. A. Benitez, M. H. Fonticelli, R. C. Salvarezza, Langmuir 2010, 18, 14655-14662.

[53] C. M. Crudden, M. Sateesh, R. Lewis, J. Am Chem. Soc. 2005, 127, 1004510050.

[54] C. M. Shen, Y. K. Su, H. T. Yang, T. Z. Yang, H. J. Gao, Chem. Phys. Lett. 2003, 373, 39-45.

[55] L. Selegard, V. Khranovskyy, F. Soderlind, C. Vahlberg, M. Ahren, P. Kall, R. Yakimova, K. Uvdal, ACS Appl. Mater.Interfaces. 2010, 2, 2128-2135.

[56] N. Huang, Y. Xu, D. Jiang, Sci. Rep. 2014, 4, 7228.

[57] T. Aditya, A. Pal, T. Pal, Chem. Commun. 2015, 51, 9410-9431.

[58] Z. Li, J. Li, J. Liu, Z. Zhao, C. Xia, F. Li, ChemCatChem. 2014, 6, $1333-$ 1339.

[59] K. L. Gares, S. V. Bykov, B. Godugu, S. A. Asher, Appl. Spectrosc. 2014, 68, 49-56.

[60] J. Noh, R. Meijboom, Appl.Surf.Sci. 2014, 230, 400-413.

[61] M. Kroger, M. E. Schumacher, H. Risse, G. Fels, Biodegradation 2004, 15, 241-248.

[62] J. Sun, Y. Fu, G. He, X. Sun, X. Wang, Catal. Sci. Technol. 2014, 4, 17421748.

[63] F. Chahdoura, C. Pradel, M. Gomez, Adv. Synth. Catal. 2013, 355, 36483660 .

[64] A. Jürgensen, N. Heutz, H. Raschke, K. Merz, R. Hergenröder, Anal. Chem. 2015, 87, 7848 .

[65] L. Chng, N. Erathodiyil, J. Y. Ying, Acc. Chem. Res. 2013, 46, 1825-1837.

Submitted: June 13, 2017

Revised: September 14, 2017

Accepted: September 15, 2017 